\title{
DESENSIBILIZACIÓN SISTEMÁTICA PARA LA FOBIA A LOS ASCENSORES
}

\section{Systematic desensitization to the phobia to elevators}

Giuliana Margarita Obregón López Investigador independiente - Lima, Perú https://orcid.org/0000-0001-5582-3109

\begin{abstract}
Resumen
El estudio de caso, aborda un paciente del sexo masculino de 19 años quien, en los últimos seis meses, ha experimentado miedo intenso e irracional a ingresar a los ascensores, en los diversos contextos de su entorno social. Como consecuencias negativas, el paciente se ha aislado progresivamente de sus amistades en la universidad, amistades del trabajo, de sus familiares y de su enamorada; con el fin de evitar usar los ascensores y ocultar la fobia que padece. En el tratamiento de intervención, se utiliza la técnica desensibilización sistemática, que permite que el paciente aprenda a exponerse a los estímulos que le generan aversión y en este caso es el utilizar el ascensor. La técnica de la desensibilización sistemática incluye el proceso de entrenamiento en relajación muscular progresiva, establecimiento de una jerarquía con las situaciones que generan ansiedad, entrenamiento en la imaginación mediante una exposición gradual de las situaciones ansiógenas y exposición en vivo a las situaciones desagradables. El proceso de intervención se desarrolla en diez sesiones. La investigación es de tipo aplicada y corresponde al diseño Univariable Multicondicional del Análisis del Comportamiento aplicado (ABA: Applied Behavior Analysis). Los resultados indican que en la línea base de pretratamiento, se obtuvo catorce conductas problemas; en comparación, a la medición de la línea postratamiento, donde las conductas problema no se presentaron. Los beneficios terapéuticos se mantuvieron después de un mes de seguimiento.
\end{abstract}

Palabras clave: Desensibilización sistemática, fobia a los ascensores.

\begin{abstract}
The case study addresses a 19-year-old male patient who, in the last six months, has experienced intense and irrational fear of entering elevators in the various contexts. As a consequence, the patient has progressively isolated himself from his friends at university, friends from work, from his relatives and from his girlfriend, in order to avoid using the elevators and hide the phobia. The systematic desensitization technique is used in the treatment. This procedure allows the patient to learn to be exposed to the stimuli that generate aversion and in this case it is to use the elevator. The systematic desensitization technique includes the process of training in progressive muscle relaxation, establishing a hierarchy with situations that generate anxiety, training in the imagination through a gradual exposure of anxiety situations and live exposure to unpleasant situations. The intervention process takes place in ten sessions. This is an applied research and uses a univariate multiconditional design of Applied Behavior Analysis (ABA). The results indicate that, in the pretreatment baseline, fourteen problem behaviors were identified; in comparison to the measurement of the post-treatment line, where the problem behaviors were not identified. Therapeutic benefits were maintained after one month of follow-up.
\end{abstract}

Keywords: Systematic desensitization, elevators phobia.

Cualquier uso que se haga de este artículo debe incluir: Autor / Título original de la publicación / ISSN.

\footnotetext{
* Articulo basado en un trabajo académico para optar al titulo de segunda especialidad profesional en Terapia Cognitivo Conductual.

** Psicóloga. giulianaobregonl@gmail.com
} 


\section{INTRODUCCIÓN}

La fobia específica de tipo situacional, se clasifica en el espectro de los trastornos de ansiedad. Este diagnóstico, se caracteriza por presentar un miedo intenso, persistente e irracional que es, provocado por la presencia de un estímulo aversivo o por la anticipación, a nivel cognitivo, de situaciones desagradables. Asimismo, según Bados (2015) el paciente generalmente evita las situaciones fóbicas; sin embargo, al exponerse al estímulo fóbico, puede soportarlo con un alto índice de ansiedad o malestar.

La fobia específica es un problema de salud pública a nivel internacional y nacional, porque mediante investigaciones científicas, se ha encontrado que las personas con fobia a los ascensores u otro tipo sin tratamiento, tienden a presentar un deterioro del desenvolvimiento psicosocial; es decir, este diagnóstico genera una alteración en el normal funcionamiento de la persona, específicamente cuando aquellos estímulos aversivos están inmersos en ámbitos de desarrollo del individuo, afectando su rendimiento laboral y académico (American Psychiatric Association, 2013).

Según Cabañas et al. (2018) en Europa, la fobia especifica está catalogada como el trastorno más frecuente de los trastornos por ansiedad y es el diagnóstico más frecuente en comparación a otros trastornos psicológicos catalogados en el Manual Diagnóstico y Estadístico de los Trastornos Mentales (DSM-V). Asimismo, la fobia especifica está relacionada con el alto índice de deterioro social y es un factor de riesgo para el desarrollo de trastornos depresivos, consumo de sustancias psicoactivas y desarrollo de fobia a otros estímulos (Delgado y Sánchez, 2019).

Los resultados de una investigación nacional realizada durante el 2005 a la población adulta, revelaron que "los tipos de trastornos con mayor incidencia, engloban a los trastornos de ansiedad (7.9 $\%)$, posteriormente los trastornos del estado de ánimo (3.5\%), los trastornos en el control de impulsos $(3,5 \%)$ y los trastornos de uso de sustancias (1,7\%)" (Piazza y Fiestas, 2014, p. 39). Cabe recalcar que, en el apartado de trastornos de ansiedad, la fobia específica presenta mayor prevalencia (4.6\%); en comparación, a la fobia social $(1.4 \%)$, a la agorafobia sin trastorno de angustia
$(0.5 \%)$, al trastorno de angustia $(0.3 \%)$, al trastorno de ansiedad generalizada $(0.2 \%)$ y al trastorno de estrés postraumático (0.2\%) (Piazza y Fiestas, 2014).

En este sentido, la presente investigación aborda un estudio de caso, de un paciente del sexo masculino de 19 años, con el diagnóstico de fobia especifica situacional, en relación al uso de ascensores. Para ello, se utiliza la técnica conductual del condicionamiento clásico, denominada desensibilización sistemática, para la disminución de los síntomas desadaptativos. El objetivo es que al finalizar el programa de tratamiento, el paciente sea capaz de utilizar los ascensores en el trabajo, la universidad, centros comerciales y en edificios departamentales; de manera individual y acompañado de diferentes personas, con niveles menores o igual a 6 en una escala del 1 al 10 de ansiedad en la Escala de Unidades subjetivas de Ansiedad - SUDS.

\section{DESENSIBILIZACIÓN SISTEMÁTICA}

La técnica desensibilización sistemática, fue diseñada por el psiquiatra Wolpe (1958, citado por Ruiz et al., 2012) para el tratamiento de las fobias y la ansiedad. Asimismo, se desarrolló un sistema de valoración cuantitativo de nivel subjetivo de malestar, denominando Escala de Unidades subjetivas de Ansiedad - SUDS (Subjective Units of Disturbance Scale) que se utiliza para jerarquizar en los pacientes el nivel de malestar producido por las situaciones aversivas (Wolpe, 1958; citado por Ruiz et al., 2012).

Mediante esta técnica el paciente aprende a enfrentar el estímulo aversivo, que en el caso expuesto es el uso del ascensor; es decir, aprende a relajarse al utilizar el ascensor. La técnica, está compuesta por el siguiente proceso: Entrenamiento en relajación progresiva de Jacobson, establecimiento de una jerarquía con las situaciones ansiógenas, entrenamiento en la imaginación mediante una exposición gradual de las situaciones ansiógenas y exposición directa a las situaciones desagradables (Ruiz et al., 2012).

\section{FOBIA ESPECÍFICA}

Capafons (2001) refiere que el miedo tiene un valor adaptativo que contribuye en la supervivencia de 
la especie humana; sin embargo, cuando esta emoción del miedo intenso se experimenta ante situaciones que no representan una amenaza se denomina fobia.

Según Bados (2015) la fobia específica se caracteriza por presentar un miedo intenso, persistente e irracional; provocado por la presencia o anticipación de situaciones concretas. Asimismo, la persona al estar expuesta al estímulo fóbico produce una respuesta inmediata de ansiedad. Estas situaciones fóbicas generalmente son evitadas; sin embargo, al exponerse al estímulo aversivo se puede soportar con un alto índice de ansiedad o malestar. Es decir, los pacientes con este diagnóstico experimentan un miedo irracional y persistente ante estímulos que no representan una verdadera amenaza. De esta manera, el individuo evita o escapa de dichos estímulos de connotación aversiva (Bados, 2015).

Según el CIE - 10 (OMS, 1992), la fobia especifica se agrupa en 3 tipos como: Fobia a los animales, fobia a la sangre-heridas y fobia situacional. En el caso de la actual investigación, el paciente presenta el diagnóstico de fobia específica de tipo situacional.

\section{Criterios diagnósticos de la fobia específica}

Para establecer el diagnóstico del estudio de caso se utilizó el CIE - 10 (OMS, 1992), en relación al apartado de Trastornos mentales y del comportamiento.

Según el CIE 10 (OMS, 1992, p.112) codifica la fobia específica como F40.2 y se encuentra dentro del apartado de trastornos de ansiedad fóbica.

A. Presencia de alguno de los siguientes:

1. Miedo marcado a un objeto o situación específicos, no incluidos en la agorafobia (F40.0) o en la fobia social (F40.1).

2. Evitación marcada de un objeto o situación específicos, no incluidos en la agorafobia (F40.0) o en la fobia social (F40.1).

3. Entre los objetos o situaciones más frecuentes están animales, pájaros, insectos, alturas, truenos, a volar en avión, espacios pequeños cerrados, la visión de sangre o heridas, inyecciones, dentistas y hospitales.
B. Los síntomas de ansiedad ante la situación temida, se deben de haber manifestado en algún momento desde el inicio del trastorno como:

1. Síntomas autonómicos (...).

2. Síntomas en el pecho y abdomen (...).

3. Síntomas relacionados con el estado mental (...).

4. Síntomas generales (...).

C. Malestar emocional significativo ocasionado por los síntomas o la evitación, y el individuo reconoce que son excesivos o irracionales.

D. Los síntomas se limitan a la situación temida o a la contemplación de la misma. fobias

Teorías explicativas sobre la adquisición de las

Condicionamiento clásico: Según Ruiz et al. (2012) para adquirir la fobia a los ascensores mediante el condicionamiento clásico, se requiere la presencia de un estímulo neutro (EN) junto a un estímulo incondicionado (EI). Cabe referir, que un estímulo neutro no produce ninguna respuesta sobre el organismo y el estímulo incondicionado produce una respuesta innata o refleja (RI). Asimismo, si el estímulo neutro y el estímulo incondicionado se presentan en simultáneo, el estímulo neutro adquiere los efectos del estímulo incondicionado; de esta manera, el organismo produce una respuesta al estímulo condicionado (EC) y esta respuesta se denomina, respuesta condicionada (RC).

Los elementos del condicionamiento clásico son los siguientes:

- Estímulo Incondicionado (EI): Es un objeto, animal o situación que produce una respuesta natural en la persona, denominado respuesta innata o refleja (Núñez et al.,2015). Por ejemplo: La oscuridad, es un estímulo incondicionado que produce miedo.

- Respuesta Incondicionada(RI): Es una respuesta natural de la persona, denominado respuesta innata o refleja, ante la presencia de un estímulo incondicionado (Núñez et al., 2015). Por ejemplo: La oscuridad (EI), produce 
una respuesta incondicionada (RI) de miedo.

- Estímulo Neutro (EN): El estímulo neutro, en un principio no genera una respuesta en el organismo. Sin embargo, cuando se presenta continuamente y en simultaneo con el estímulo incondicionado, adquiere las funciones del estímulo incondicionado (Núñez et al., 2015). Por ejemplo: En el presente caso, es el ascensor.

- Estímulo Condicionado (EC): Inicialmente fue un estímulo neutro, que adquirió las funciones del estímulo incondicionado (Núñez et al., 2015). Por ejemplo: En el caso expuesto, es el ascensor.

- Respuesta Condicionada (RC): Es una respuesta o conducta aprendida, que se genera ante la exposición de un estímulo condicionado (Núñez et al., 2015). Por ejemplo: En el caso expuesto es el miedo al ascensor.

Según lo expuesto sobre la adquisición de la fobia mediante el condicionamiento clásico, se puede inferir que la fobia a los ascensores se adquiere de la siguiente manera, en el presente caso: La oscuridad representa un estímulo incondicionado (EI), que genera una respuesta incondicionada de miedo (RI), y a su vez el paciente ingresa a un ascensor oscuro; es así que, luego de varios apareamientos entre el estímulo incondicionado (oscuridad) y el estímulo neutro que vendría a ser el ascensor, la persona manifiesta una respuesta condicionada de miedo (RC) ante el estímulo condicionado (EC) representado por el ascensor.

Condicionamiento operante: Según O'Donohue y Fisher (2012) la conducta fóbica al igual que otros comportamientos disfuncionales, también se adquiere y mantiene a través de la triple relación de contingencia que está constituida por tres elementos denominados: Antecedentes(A), comportamiento(B) y consecuencias (C). Cabe referir, que los antecedentes son estímulos externos (eventos ambientales, situaciones, objetos, personas etc.) o internos (pensamientos, recuerdos etc.) que se presentan de manera constante en el contexto y genera un comportamiento o respuesta. Asimismo, dependiendo del tipo de contingencia, la consecuencia aumenta o disminuye la probabilidad de que en el futuro el comportamiento se emita en presencia de antecedentes similares.
A partir de lo expuesto, se realiza un ejemplo en un caso de fobia a los ascensores, donde la conducta se ha adquirido y se mantiene por condicionamiento operante: Un joven al tener que usar el ascensor (antecedente), prefiere utilizar las escaleras (comportamiento) y experimenta alivio (consecuencias). Asimismo, las consecuencias en este caso aumentan la probabilidad que, posteriormente el comportamiento se emita en presencia de antecedentes similares.

Aprendizaje vicario o social: Sapp (2004) sustenta que las teorías del aprendizaje social integran teorías cognitivo conductuales y sociales, en las que el individuo es visto como un actor que es influenciado por la interacción de factores interpersonales, conductuales, cognitivas, ambientales y sociales. Este enfoque propone que, el individuo puede desarrollar fobias cuando observa a personas con conductas fóbicas ante un determinado estímulo y por observar que dicho estímulo genera accidentes peligrosos a otras personas (Milosevic y Mc Cabe, 2015).

Este aprendizaje se adquiere; por ejemplo: Cuando un joven al observar en la televisión una noticia sobre el fallecimiento de un trabajador, el cual fue aplastado por el ascensor mientras limpiaba el primer compartimiento. En ese momento, el joven piensa "los ascensores son muy peligrosos y podemos morir ahí adentro, porque siempre se malogran". De este modo, el joven aprende que el utilizar los ascensores, son un peligro para su vida.

Teoría biológica: Bados (2015) refiere que algunos de los integrantes de las familias con diagnóstico de fobias específicas tienden a adquirir la vulnerabilidad ansiosa, por la carga genética de hipersensibilidad al estrés; sin embargo, no se hereda genéticamente la fobia al mismo estímulo temido. Cabe referir que, Las fobias específicas se pueden desarrollar en personas sin condiciones de predisposición biológica, cuando las experiencias negativas (por procesos de condicionamiento clásico, operante o vicario) han sido suscitadas (Bados, 2015). Por último, las fobias específicas en general, se adquieren y mantienen mediante una interacción entre los factores biológicos, psicológicos y sociales (Bados, 2015). 


\section{MÉTODO}

\section{Tipo y diseño de investigación}

Investigación de tipo Aplicada: El estudio de caso, se basa en la terapia conductual que, a través de experimentos, ha comprobado su efectividad en dar soluciones prácticas, mediante técnicas de intervención psicológicas, a una necesidad social (Vargas, 2009).

Diseño Univariable Multicondicional ABA: Se denomina Univariable, porque se maneja una sola Variable Independiente; y en el caso expuesto la Variable Independiente es la técnica desensibilización sistemática. El aspecto Multicondicional, hace referencia a la presencia de dos o más fases experimentales; es decir, a la fase A, B y A. De esta manera, se establece la línea base, sin tratamiento o intervención (A); para luego, implementar la fase B, aplicándose el tratamiento y la medición de frecuencias de las conductas desadaptativas. Finalmente, se retira el tratamiento y se vuelve a medir la frecuencia de las conductas desadaptativas (A) (Grinnell, Williams y Unrau, 2009, citado por Hernández, Fernández y Baptista, 2014).

\section{DESCRIPCIÓN DEL CASO}

\section{Identificación del paciente}

Nicolás (nombre figurado) es un joven de 19 años de edad y estudiante universitario de la carrera de administración. Proviene de una familia nuclear, presenta mayor afinidad hacia la madre y escasa confianza con el padre. Es el menor de dos hermanos, $\mathrm{y}$ vive con sus padres.

\section{Motivo de consulta}

El Paciente refiere "tengo un terrible miedo a los ascensores, de diferentes lugares, desde hace más de seis meses y eso me está causando problemas". "También, estoy trabajando hace dos meses en una oficina que queda en el noveno piso y para llegar a la oficina tengo que subir por las escaleras y estoy llegando tarde o cansado".

\section{Problema Actual}

El paciente presenta ansiedad, miedo intenso e irracional cuando tiene que utilizar los ascensores en su centro laboral, universidad, centros comerciales y departamentos. A continuación, se detallan los sucesos:

Nicolás, cuando está a 6 metros del ascensor del primer piso, del edificio de su trabajo, piensa "No quiero utilizar el ascensor porque se va a malograr y no podré salir, me quedaré encerrado ahí dentro y sin poder respirar" experimentando miedo (10/10) y ansiedad (10/10), sudoración palmar, al momento de sentir una sensación de calor sus mejillas se sonrojan y siente que se ahoga. A lo cual opta por alejarse del ascensor y subir por las escaleras hasta el noveno piso y los síntomas van desapareciendo, experimentando tranquilidad, alivio, desaparece la sudoración y la sensación de ahogo. Esta conducta se da cada vez que Nicolás asiste al trabajo, aproximadamente 5 veces por semana.

Cuando Nicolás se encuentra en la universidad y debe asistir al salón de clases que se encuentra en décimo piso, al cual va a llegar con retraso, piensa "otra vez voy a llegar tarde porque voy a tener que subir por las escaleras, pero mejor así porque si voy al ascensor me voy a sentir muy mal". Experimenta ansiedad (10/10), frustración (10/10), empieza a experimentar sudoración palmar, tensión muscular y su rostro se sonroja. Ante ello, Nicolás usa las escaleras, disminuyendo en intensidad las emociones experimentadas previamente, aliviándose las respuestas fisiológicas y desapareciendo los pensamientos anteriormente mencionados. Esta conducta se presenta cuando Nicolás debe subir a su salón de clases que está en décimo piso, esto ocurre aproximadamente 5 veces a la semana.

En otro momento, Nicolás al estar en un centro comercial con sus amigos y tienen que utilizar el ascensor, piensa "ahora mis amigos se van a burlar de mí porque soy un miedoso" y experimenta vergüenza $(10 / 10)$, miedo (10/10), ansiedad (10/10), empieza a experimentar sudoración palmar, tensión muscular, su rostro se sonroja y siente que su cuerpo tiembla; a lo cual, sus amigos empiezan a burlarse diciéndole "Cómo vas a tenerle miedo a los ascensores", "Que miedoso que eres" y lo empujan en dirección al ascensor. Para ello, Nicolás siente cólera $(8 / 10)$ y opta por subir por las escaleras hasta el cuarto piso, empezando a experimentar alivio. Esta conducta se presenta cuando 
Nicolás sale con sus amigos, aproximadamente $1 \mathrm{vez}$ por semana.

Nicolás al ir al departamento de su enamorada y al estar a 3 metros del ascensor del primer piso, piensa: "Mi enamorada creerá que soy un cobarde, porque tengo miedo a los ascensores", y experimenta vergüenza (8/10), miedo (9/10), ansiedad (8/10), sudoración palmar, tensión corporal y dolor de cabeza; por ello, decide subir por las escaleras hasta el piso diecisiete y empieza a experimentar alivio, disminución de la sudoración palmar, tensión corporal y dolor de cabeza. Esta conducta se da cada vez que Nicolás visita a su enamorada, aproximadamente 2 veces por semana.

Cuando Nicolás llega al edificio del departamento de sus tíos, quienes viven en el piso quince, al estar a un metro del ascensor, piensa: "Este ascensor es antiguo y me quedaré atrapado", experimentando miedo (9/10), ansiedad (9/10), impotencia (9/10) tensión muscular, dolor de cabeza y sudoración palmar. Ante ello, la ansiedad de Nicolás se incrementa y decide subir por las escaleras hasta el piso quince, experimentado alivio. Esta conducta se presenta aproximadamente 1 vez por semana.

\section{Historia del problema}

A la edad de 13 años, Nicolás en compañía de su madre fueron a una galería antigua y al momento de subir al ascensor en dirección al cuarto piso, el ascensor se detiene, las luces se apagan y la puerta se mantiene cerrada por 15 minutos. Para ello, Nicolás pensó "el ascensor se va a caer y vamos a morir", empezó a golpear las puertas, sintió miedo (10/10) y ansiedad (10/10); experimentó sudoración corporal, sensación de ahogo, taquicardia y temblores en las extremidades inferiores; y la madre empezó a golpear las puertas y gritando pedía auxilio.

A la edad de 16 años, el paciente visualizó una noticia nacional referente a un fallecimiento de un joven trabajador en una galería y fue aplastado por el ascensor mientras limpiaba el primer compartimiento. En ese momento, el paciente pensó "los ascensores son muy peligrosos y podemos morir ahí adentro, porque siempre se malogran" y empezó a buscar en internet los accidentes que ocurrían producto de los ascensores, sintió miedo (8/10) y ansiedad (8/10), experimentó sudoración palmar, temblores en las extremidades inferiores y sensación de sofocación.

A la edad de 18 años, Nicolás visitó un centro comercial con sus primos y al momento de ingresar al ascensor sus primos lo empujan hacia adentro, en ese momento experimentó miedo (10/10) vergüenza (10/10), sensación de ahogo, taquicardia, y temblores en las extremidades inferiores; para ello, inmediatamente se escapó del ascensor y experimentó alivio.

\section{Evaluación}

Mediante la evaluación psicológica se utilizó la entrevista y observación conductual. Para establecer el diagnóstico, se usó los criterios del CIE-10. Asimismo, se realizó el diagnóstico funcional y análisis funcional.

Los instrumentos psicométricos utilizados, fueron los siguientes:

Cuestionario de Personalidad de Eysenck (CPE), adaptado a Lima por Araujo (2000, citado por Torres, 2016): Nicolás, presenta características de personalidad introvertida, es decir, se muestra retraído socialmente, reservado, prefiere las actividades intelectuales, evita aquellas actividades que impliquen una participación social activa y le cuesta establecer relaciones interpersonales con mujeres de su edad que recién conoce. El paciente usualmente se aleja de sus amigos, para evitar que se percaten y se burlen de él, en relación al miedo que le causa usar los ascensores. Respecto a sus emociones, presenta un elevado nivel de ansiedad, inseguridad y miedo, emitiendo comportamientos de evitación y escape frente a estímulos aversivos para él como, por ejemplo, usar el ascensor. El miedo intenso que presenta al uso de los ascensores, interfiere en su desenvolvimiento social, porque le afecta emocionalmente, que algunos parientes y amigos se burlen de su condición. Finalmente, muestra adaptación a las reglas sociales y familiares.

Test de inteligencia para adultos de Wechsler (WAIS III): Presenta un Coeficiente Intelectual total de 108, situándose en el Nivel Intelectual Promedio, en comparación a otros sujetos de su edad; es decir, posee la capacidad para comprender aspectos complejos y 
resolver complicaciones a través del razonamiento. Asimismo, presenta un Coeficiente Intelectual Verbal de 104, y se ubica en el Nivel correspondiente a Promedio, en comparación a otros sujetos de su edad. También, presenta un Coeficiente Intelectual Ejecutivo de 111 y se ubica en el Nivel Promedio Alto, en comparación a otros sujetos de su edad.

\section{Intervención psicológica}

El tratamiento psicológico, se desarrolló en diez sesiones, de 60 minutos por sesión y una vez por semana, durante aproximadamente 3 meses.

\section{Objetivos terapéuticos}

Objetivos General:

$\mathrm{Al}$ finalizar el programa de tratamiento, el joven será capaz de utilizar los ascensores en el trabajo, la universidad, centros comerciales y en edificios departamentales; de manera individual y acompañado de diferentes personas, con niveles menores o igual a 6 en una escala del 1 al 10 de ansiedad.

\section{Procedimiento}

La intervención psicológica incluye un mismo procedimiento para los 5 objetivos específicos porque la a conducta problema es subir por las escaleras para evitar el uso del ascensor, en distintos escenarios.

\section{Paso 1: Técnica psicoeducación.}

Constituye un elemento básico para el desarrollo de la intervención porque a través de esta técnica se explica e informa en qué consiste el tratamiento, las características del diagnóstico clínico y cuáles son los objetivos que se pretenden alcanzar durante el proceso psicoterapéutico (Guerra y Plaza, 2009).

En el caso expuesto, se explica al paciente las diferencias entre el miedo adaptativo y el miedo fóbico, en qué consiste la fobia a los ascensores, cuáles son los síntomas en relación a lo que el paciente experimenta y las consecuencias de seguir manteniendo este problema. Asimismo, se indica los objetivos que se pretender alcanzar, durante la psicoterapia.

\section{Paso 2: Técnica desensibilización sistemática}

a) Se realiza el entrenamiento de la técnica Relajación Muscular Progresiva breve de Jacobson:
Se explica al paciente los beneficios de la técnica de relajación; mediante la cual, logrará disminuir considerablemente la tensión fisiológica ante situaciones desagradables y en este caso relacionado al uso del ascensor. De esta manera, cuando sienta tensión muscular en alguna parte de su cuerpo por el estímulo desagradable, tendrá la capacidad de relajar sus músculos y disminuir la tensión (Ruiz et al., 2012). Se trabaja con cuatro principales grupos musculares sentado en una silla:

- Músculos de la mano, antebrazo y el bíceps: El paciente cierra ambos puños, tensando los bíceps y los antebrazos, por 5 a 7 segundos y después relajarse 20 a 30 segundos. Asimismo, se observa la diferencia entre la sensación de tensión y de relajación. Y se repite este proceso 3 veces (Ruiz et al., 2012).

- Músculos de la cabeza, cara, cuello y hombros: Se le indica que arrugue la frente; mueva la cabeza hacia atrás formando un círculo, arrugue los músculos de la cara y encoja los hombros; por 5 a 7 segundos y después relajarse 20 a 30 segundos. Se observa la diferencia entre la sensación de tensión y de relajación. Y se repite este proceso 3 veces (Ruiz et al., 2012).

- Músculos del tórax, de la región del estómago y de la región lumbar: Separe los hombros arqueando la espalda durante 5 a 7 segundos y relajarse de 20 a 30 segundos. Hacer una inspiración profunda apretándose levemente la región del estómago con la mano, sostenga la respiración 5 segundos y suelte la respiración relajándose (Ruiz et al., 2012).

- Músculos del muslo de las nalgas, pantorrillas y pies: Extiende los pies y los dedos, dirigiendo las puntas hacia arriba fuertemente para tensar durante 5 a 7 segundos y se relaja de 20 a 30 segundos. Finalmente, flexione los dedos tensando la pantorrilla, los muslos y las nalgas, durante 5 a 7 segundos y se relaja de 20 a 30 segundos (Ruiz et al., 2012).

b) Se establece una jerarquía, mediante una graduación de las situaciones ansiógenas en el proceso de utilizar el ascensor (Ruiz et al., 2012). La jerarquía se detalla en la tabla 1. 


\section{Tabla 1}

Jerarquización de situaciones aversivas

Estar en la puerta del ascensor, del primer piso.

Subir al segundo piso en el ascensor, acompañado por la terapeuta.

Bajar del segundo piso al primer piso en el ascensor, acompañado por la terapeuta.

Subir del primer piso al décimo piso en el ascensor, acompañado por la terapeuta.

Bajar del décimo piso al primero piso, acompañado por la terapeuta.

Subir del primer piso al piso diecisiete, acompañado por la terapeuta.

Bajar del piso diecisiete al primer piso, acompañado por la terapeuta.

Subir al segundo piso en el ascensor, solo.

Bajar del segundo piso al primer piso en el ascensor, solo.

Subir del primer piso al décimo piso en el ascensor, solo.

Bajar del décimo piso al primero piso en el ascensor, solo.

Subir del primer piso al piso diecisiete en el ascensor, solo.

Bajar del piso diecisiete al primer piso en el ascensor, solo.

Nota: SUDS = Escala de unidades subjetivas de ansiedad.

c) Se realiza el Entrenamiento en la Imaginación, es decir, de manera progresiva se pide al paciente imaginar las situaciones de la jerarquía, que le generan ansiedad, empezando por la situación con menor puntaje, que es "estar en la puerta del ascensor, del primer piso". Considerando que cada exposición imaginaria del paciente a la situación ansiógena, se mantendrá durante 20 segundos aproximadamente, experimentando la ansiedad y luego se va a centrar en la relajación durante 50 segundos. Finalmente, cuando el estímulo temido no genere ansiedad, se volverá a exponer en la imaginación tres veces consecutivas (Ruiz et al., 2012).

d) Se acompaña al paciente a un ascensor para iniciar con el procedimiento de Exposición en Vivo ante las situaciones ansiógenas, iniciando por la jerarquía con menor puntaje que es "estar en la puerta del ascensor, del primer piso". Se considera que cada exposición del paciente a la situación ansiógena, en relación a la jerarquía, se mantendrá durante 20 segundos aproximadamente, experimentando la ansiedad y luego se va a centrar en la relajación durante 50 segundos (Ruiz et al., 2012).
Cuando una situación de la jerarquía con menor puntaje no genere la respuesta de ansiedad, se vuelve a exponer al paciente tres veces consecutivas a dicha situación. De esta manera, se continúa con las siguientes situaciones en un orden progresivo, de menor ansiedad a mayor ansiedad (Ruiz et al., 2012).

\section{Paso 3: Utilizar la técnica reforzamiento positivo}

La técnica del reforzamiento positivo, consiste en incrementar la frecuencia de una conducta, a la que procede un evento u objeto agradable para la persona, denominado reforzador positivo (Ruiz et al., 2012). Por ejemplo: Cada vez que el paciente cumple con el objetivo de utilizar el ascensor, se le premia con halagos como "lo hiciste muy bien", "me siento muy orgullosa de ti" y se le entrega una galleta de su agrado.

\section{Paso 4: Tareas para la casa.}

Ruiz et al. (2012) mencionan que, las instrucciones como tareas para la casa, tienen por finalidad generalizar las conductas aprendidas durante las sesiones terapéuticas; es decir, mantener los nuevos aprendizajes o respuestas adaptativas y transferir el entrenamiento a otros contextos o 
escenarios. Asimismo, estas instrucciones se brindan cuando el paciente alcanza un óptimo desarrollo en el entrenamiento de una habilidad. Además, se debe considerar que, al iniciar las sesiones terapéuticas, las tareas para la casa mostraran accesibilidad y sencillez.

En el caso expuesto como instrucciones de tareas para la casa, se indica al paciente lo siguiente:

- Practicar la relajación muscular progresiva cinco veces a la semana; durante 15 minutos como mínimo, por día.

- Practicar a nivel imaginario la exposición ante el estímulo temido, utilizando la técnica relajación muscular progresiva. Este proceso se debe realizar aproximadamente 20 minutos por día y cinco veces a la semana.
- Al alcanzar la relajación ante el estímulo temido en la imaginación, practicar la exposición en vivo y utilizar la relajación ante indicadores de tensión corporal. Realizar este proceso cada vez que se expone al estímulo temido, es decir cada vez que utiliza el ascensor.

\section{RESULTADOS}

La línea base pretratamiento (A) se estableció en la semana 1 (ver tabla 2 y figura 1 ). La línea de tratamiento (B) se ejecutó de la semana 2 a la semana 10 y la línea de seguimiento (A) se desarrolló en la semana 14 (ver tabla 3 y figura 2).

\section{Tabla 2}

Registro de frecuencia de la conducta motora, en la semana 1, denominada línea base

\begin{tabular}{|c|c|c|c|c|c|c|c|c|}
\hline Conductas & $\mathrm{L}$ & M & M & $\mathrm{J}$ & $\mathrm{V}$ & $\mathrm{S}$ & $\mathrm{D}$ & Total \\
\hline $\begin{array}{l}\text { A. Subir por las escaleras hasta el noveno piso, } \\
\text { del edificio del trabajo, para evitar usar el ascensor. }\end{array}$ & I & I & I & I & $\mathrm{I}$ & - & - & 5 \\
\hline $\begin{array}{l}\text { B. Subir por las escaleras hasta el décimo piso, del } \\
\text { aula de la universidad, para evitar usar el ascensor. }\end{array}$ & I & I & I & I & I & - & - & 5 \\
\hline $\begin{array}{l}\text { C. Subir por las escaleras hasta el cuarto piso, } \\
\text { de un centro comercial, para evitar usar el ascensor. }\end{array}$ & - & - & - & - & I & - & - & 1 \\
\hline $\begin{array}{l}\text { D. Subir por las escaleras hasta el piso diecisiete, } \\
\text { del departamento de su enamorada, } \\
\text { para evitar usar el ascensor. }\end{array}$ & - & - & - & - & - & I & I & 2 \\
\hline $\begin{array}{l}\text { E. Subir por las escaleras hasta el piso quince del } \\
\text { departamento de sus tíos, para evitar usar el ascensor. }\end{array}$ & - & - & - & - & - & - & I & 1 \\
\hline
\end{tabular}

Nota: $\mathrm{L}=$ Lunes; $\mathrm{M}=$ Martes; $\mathrm{M}$ = Miércoles; $\mathrm{J}=$ Jueves; $\mathrm{V}=$ Viernes; $\mathrm{S}=$ Sábado; $\mathrm{D}=$ Domingo. 


\section{Figura 1}

Registro de frecuencia de la conducta motora, en la semana 1, denominada línea base

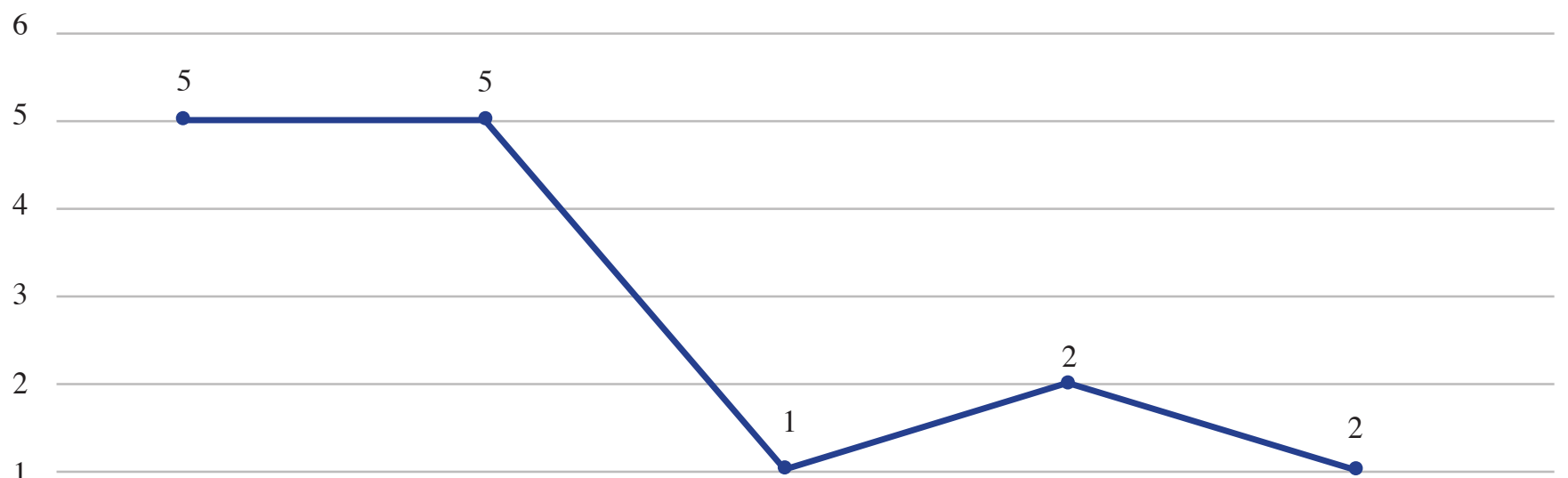

0
Conducta A
Conducta B
Conducta C
Conducta D
Conducta $\mathrm{E}$

Semana 1

Tabla 3

Registro de frecuencia de la conducta motora durante las semanas de tratamiento

\begin{tabular}{|c|c|c|c|c|c|c|}
\hline Conductas & Semana 01 & Semana 02 & Semana 06 & Semana 08 & Semana 10 & Semana 14 \\
\hline $\begin{array}{l}\text { A. Subir por las escaleras hasta el } \\
\text { noveno piso, del edificio del trabajo, } \\
\text { para evitar usar el ascensor. }\end{array}$ & 5 & 5 & 3 & 0 & 0 & 0 \\
\hline $\begin{array}{l}\text { B. Subir por las escaleras hasta el } \\
\text { décimo piso, del aula de la universidad, } \\
\text { para evitar usar el ascensor. }\end{array}$ & 5 & 5 & 2 & 1 & 0 & 0 \\
\hline $\begin{array}{l}\text { C. Subir por las escaleras hasta el cuarto } \\
\text { piso, de un centro comercial, } \\
\text { para evitar usar el ascensor. }\end{array}$ & 1 & 1 & 0 & 0 & 0 & 0 \\
\hline $\begin{array}{l}\text { D. Subir por las escaleras hasta el piso } \\
\text { diecisiete, del departamento de su } \\
\text { enamorada, para evitar usar el ascensor. }\end{array}$ & 2 & 2 & 2 & 0 & 0 & 0 \\
\hline $\begin{array}{l}\text { E. Subir por las escaleras hasta el piso } \\
\text { quince del departamento de sus tíos, } \\
\text { para evitar usar el ascensor. }\end{array}$ & 1 & 1 & 1 & 0 & 0 & 0 \\
\hline
\end{tabular}




\section{Figura 2}

Registro de frecuencia de la conducta motora durante las semanas de tratamiento

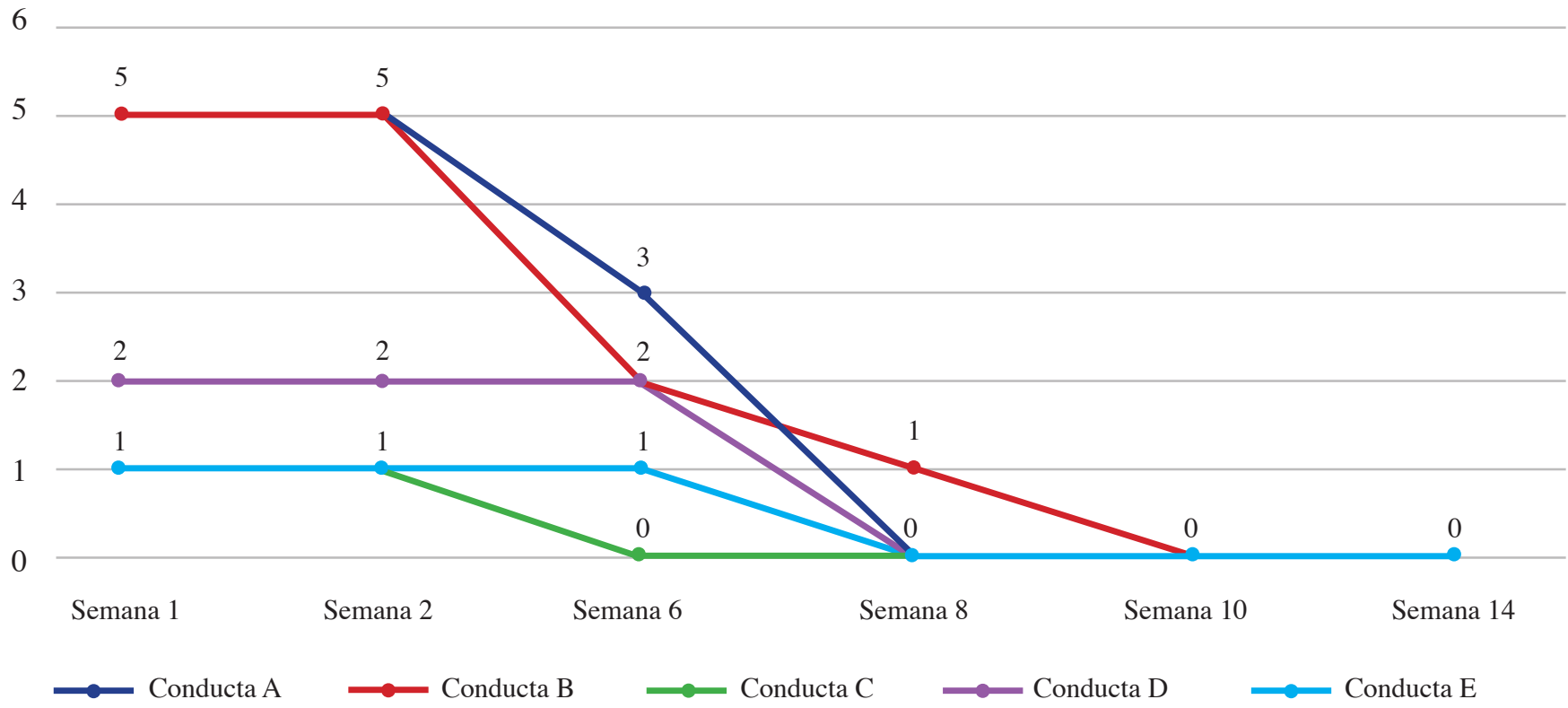

Resultados generales: Como se observa en la línea base de pretratamiento (A) (Figura 1) en la semana 1, se obtuvo de manera general 14 conductas problema en relación a usar las escaleras en distintos contextos para evitar usar el ascensor (ver tabla 2). Durante la aplicación del tratamiento, las frecuencias de las conductas problema disminuyeron sustancialmente, en comparación a la línea base (ver Tabla 3). (Figura 2) Posteriormente, en la medición de la línea de seguimiento (A), correspondiente a la semana 14 , se observa el mantenimiento de logros y las conductas problema no se presentaron (ver Tabla 3). Mediante estos resultados se corrobora la efectividad de la técnica utilizada dentro del programa. Por último, el joven fue capaz de utilizar los ascensores en el trabajo, la universidad, centros comerciales y en edificios departamentales; de manera individual y acompañado de diferentes personas, con niveles menores o igual a 6 , en una escala del 1 al 10 de ansiedad.

La conducta problema (a) Subir por las escaleras hasta el noveno piso, del edificio del trabajo, para evitar usar el ascensor: En la línea base de pretratamiento (A)
(Figura 1) se observa que en la semana 1, la conducta problema se presentó con una frecuencia de 5 veces y en la semana 2 que se inicia la línea de tratamiento (B) (Figura 2) se mantuvo estable con la misma frecuencia. En la semana 6 la conducta problema se presentó 3 veces, disminuyendo ligeramente en comparación con la línea base. Esta disminución se hizo aún más evidente en la semana 8 y 10 , donde no se presentó dicha conducta problema. Durante la medición de la línea de seguimiento (A), correspondiente a la semana 14 , se observa un mantenimiento de los resultados de la fase de tratamiento; es decir, la conducta problema no se presentó, corroborándose los efectos esperados del tratamiento (ver Tabla 3). De esta manera, los resultados indican que el paciente logró utilizar el ascensor para dirigirse al noveno piso del edificio del trabajo.

La conducta problema (b) Subir por las escaleras hasta el décimo piso, del aula de la universidad, para evitar usar el ascensor: En la línea base de pretratamiento (A) (Figura 1) se observa que en la semana 1, la conducta problema se presentó con una 
frecuencia de 5 veces y en la semana 2 que se inicia la línea de tratamiento (B) (Figura 2) se mantuvo estable con la misma frecuencia. En la semana 6 la conducta problema se presentó 2 veces, disminuyendo ligeramente en comparación con la línea base. Esta disminución se hizo más evidente a partir de la semana 8 , en donde la conducta problema se presentó una vez y en la semana 10 la conducta problema no se presentó. Durante la medición de la línea de seguimiento (A), correspondiente a la semana 14, se observa un mantenimiento de los resultados de la fase de tratamiento; es decir, la conducta problema no se presentó, corroborándose los efectos esperados del tratamiento (ver Tabla 3). De esta manera, los resultados indican que el paciente logró utilizar el ascensor para llegar al décimo piso, del aula de la universidad.

La conducta problema (c) Subir por las escaleras hasta el cuarto piso, de un centro comercial, para evitar usar el ascensor: En la línea base de pretratamiento (A) (Figura 1) se observa que en la semana 1, la conducta problema se presentó una vez y en la semana 2 que se inicia la línea de tratamiento (B) (Figura 2) se mantuvo estable con la misma frecuencia. En la semana 6, 8 y 10 la conducta problema no se presentó, mostrándose una disminución en comparación con la línea base. Durante la medición de la línea de seguimiento (A), correspondiente a la semana 14 se observa un mantenimiento de los resultados de la fase de tratamiento; es decir, la conducta problema no se presentó, corroborándose los efectos esperados del tratamiento (ver Tabla 3). Por ende, los resultados indican que el paciente logró utilizar el ascensor acompañado por sus amigos, para llegar al cuarto piso, de un centro comercial.

La conducta problema (d) Subir por las escaleras hasta el piso diecisiete, del departamento de su enamorada, para evitar usar el ascensor: En la línea base de pretratamiento (A) (Figura 1) se observa que en la semana 1, la conducta problema se presentó 2 veces, en la semana 2 que se inicia la línea de tratamiento (B) (Figura 2) y semana 6 se mantuvo estable con la misma frecuencia. En la semana 8 y 10 la conducta problema no se presentó, mostrándose una disminución considerable en comparación con la línea base. Durante la medición de la línea de seguimiento (A), correspondiente a la semana 14 , se observa un mantenimiento de los resultados de la fase de tratamiento; es decir, la conducta problema no se presentó, corroborándose los efectos esperados del tratamiento (ver Tabla 3). En síntesis, los resultados indican que el paciente logró utilizar el ascensor para llegar al piso diecisiete, del departamento de su enamorada.

La conducta problema (e) Subir por las escaleras hasta el piso quince, del departamento de sus tíos, para evitar usar el ascensor: En la línea base de pretratamiento (A) (Figura 1) se observa que en la semana 1, la conducta problema se presentó una vez, en la semana 2 que se inicia la línea de tratamiento (B) (Figura 2) y en la semana 6 se mantuvo estable con la misma frecuencia. En la semana 8 y 10 la conducta problema no se presentó, mostrándose una disminución en comparación con la línea base. Durante la medición de la línea de seguimiento (A), correspondiente a la semana 14 , se observa un mantenimiento de los resultados de la fase de tratamiento; es decir, la conducta problema no se presentó, corroborándose los efectos esperados del tratamiento (ver Tabla 3 ). De esta manera, los resultados indican que el paciente logró utilizar el ascensor, para llegar al piso quince del departamento de sus tíos.

\section{DISCUSIÓN}

Los resultados del presente estudio indican que, al aplicar la variable independiente, denominada desensibilización sistemática, sobre la variable dependiente, que es la fobia a los ascensores se genera una disminución de las conductas disfuncionales en torno a la misma. Por ende, se concluye que se ha conseguido el objetivo general de la intervención psicoterapéutica. Es decir, al aplicar la técnica desensibilización sistemática, el paciente fue capaz de utilizar el ascensor en el trabajo, la universidad, centros comerciales y en edificios departamentales, de manera individual y acompañado de diferentes personas, con niveles menores o igual a 6 en una escala del 1 al 10 de ansiedad, en diez ensayos consecutivos.

Alarcón (2013) refiere que la validez interna de las investigaciones experimentales en psicología, se basan en los cambios producidos en la variable 
dependiente, como efecto específico de la variable independiente. Para ello, el experimento debe realizarse en condiciones de riguroso control, para evitar la intromisión de variables extrañas. De esta manera, en la actual investigación a través del control experimental denominado validez interna, que incluye el aislamiento de variables extrañas y el adecuado manejo de la manipulación de la variable independiente, sobre la variable dependiente, se contribuye en demostrar la efectividad de la técnica desensibilización sistemática en el tratamiento de la fobia a los ascensores. Poniendo en énfasis que, el adecuado uso de las variables son condiciones fundamentales en diseños experimentales de sujeto único.

Los resultados de la presente investigación indican que, en la línea base de pretratamiento, se obtuvo de manera general 14 conductas problema; durante la aplicación del tratamiento, la frecuencia de las conductas problema disminuyeron sustancialmente; y en la medición de la línea base postratamiento, donde el programa fue suspendido, las conductas problema no se presentaron, al igual que en la fase de seguimiento. Mediante estos resultados se corroboran la efectividad de la técnica utilizada dentro del programa. De esta manera, los resultados del presente estudio, muestran consistencia en comparación a otras investigaciones, que a nivel experimental han comprobado su efectividad en programas de intervención, mediante el uso de la técnica desensibilización sistemática, para el tratamiento de fobias específicas (Bados, 2015; Delgado y Sánchez, 2019).

Sandi (2019) realizó una investigación denominada "Desensibilización sistemática en una mujer adulta con fobia a los gatos" en Lima. La variable dependiente de estudio estuvo relacionada a la fobia a los gatos y la técnica de modificación conductual empleada fue la desensibilización sistemática. Los resultados del investigador indican que, la paciente logró exponerse a las situaciones temidas, con niveles de ansiedad menores o igual a 6 de 10, en el nivel de la escala de unidades subjetivas de ansiedad (SUDS); es decir, la paciente logró exponerse al estímulo aversivo con niveles bajos de ansiedad. En comparación a la presente investigación, también se utilizó la técnica de desensibilización sistemática, en un proceso de 10 sesiones de intervención psicológica y el paciente
Nicolás (nombre figurado) logró utilizar el ascensor en distintitos contextos con niveles menores a 6 de 10 , de la escala de unidades subjetivas de ansiedad (SUDS). Por ende, ambos resultados muestran similitud.

Escudero (2017) desarrolló una investigación titulada "Tratamiento Cognitivo Conductual en un caso de Fobia a la Sangre, Inyecciones y/o Heridas" en Madrid. Se propuso como objetivo describir, desarrollar y valorar el proceso terapéutico en una paciente de 31 años con fobia a la sangre, inyecciones $\mathrm{y} / \mathrm{o}$ heridas. El programa terapéutico estuvo compuesto por 17 sesiones, con una hora de duración, durante 3 meses. Las técnicas utilizadas fueron la psicoeducación, exposición en imaginación y en vivo con tensión aplicada, entrenamiento en tensión muscular, entrenamiento en control de la respiración, entrenamiento en habilidades de expresión, reestructuración cognitiva, detección del pensamiento, autoinstrucciones y activación conductual. Los resultados mostraron un efecto positivo en el tratamiento y el alcance de los objetivos propuestos en el programa terapéutico. A diferencia de la presente investigación, donde se puso énfasis en el uso de técnicas conductuales, elegidas en función a la problemática que presentaba el paciente y por sustento de investigaciones experimentales, en las cuales recomiendan el uso de técnicas derivadas del principio del contracondicionamiento para el caso de miedos, fobias o ansiedad (Ruiz et al., 2012). En similitud, con el estudio de Escudero (2017) y la actual investigación, en ambos resultados se obtuvieron efectos beneficiosos en el tratamiento de los pacientes.

\section{REFERENCIAS}

Alarcón, R. (2013). Métodos y diseños de investigación del comportamiento. (2da. Ed.). Fondo Editorial Ricardo Palma.

American Psychiatric Association (APA). (2013). Manual Diagnóstico y Estadístico de los Trastornos Mentales DSM-V. Masson.

Bados López, A. (2015). Fobias específicas. En M. Vallejo Pareja (Coord.), Manual de terapia de conducta (pp. 191-253). Dykinson. 
Cabañas, V., Casanova, I., \& Fernández, I. (2018). Terapia cognitivo conductuales en un caso único de fobia a la conducción. Revista de Casos Clínicos en Salud Mental, 6 (1), 71-83. https://dialnet. unirioja.es/servlet/articulo?codigo $=6642693$

Capafons, B., J. (2001). Tratamientos psicológicos eficaces para las fobias específicas. Psicothema. 1 (3), 447-452. https://bit.ly/3n1161x

Delgado, A. \& Sánchez, J. (2019). Miedo, fobias y sus tratamientos. Revista Electrónica de psicología Iztacala, 22 (2), 798-833. https://www. medigraphic.com/pdfs/epsicologia/epi-2019/ epi192c.pdf

Escudero, M. B. (2017). Tratamiento Cognitivo Conductual en un caso de Fobia a la Sangre, Inyecciones y/o Heridas. (Tesis de maestría). Universidad Autónoma de Madrid.

Guerra, V. \& Plaza, H. (2009). Tratamiento cognitivo - conductual del estrés post traumático en un caso de violación infantil. Revista de Psicología de la Universidad de Chile. XVIII (1), 103-129. https:// bit.ly/3vjgCXO

Hernández, C., Fernández, C. \& Baptista, P. (2014). Estudios de caso. En Hernández, C., Fernández, C. \& Baptista, P. (Edits.), Metodología de la investigación. (pp. 1-35). Mc Graw-Hill.

Milosevic, I., \& Mc Cabe, R. (Eds.). (2015). Phobias. The Psychology of Irrational Fear [Fobias. La Psicología del Miedo Irracional]. Greenwood.

Núñez, M., Sebastián, A. \& Muñoz, D. (2015). Principios de condicionamiento clásico de Pavlov en la estrategia creativa publicitaria. Opción, (2), 813-831. https://www.redalyc.org/ pdf/310/31045568044.pdf

Organización Mundial de la Salud. (1992). CIE-10. Décima revisión de la clasificación internacional de las enfermedades. Trastornos mentales y del comportamiento. Descripción clínica y pautas para el diagnóstico. MÉDITOR.

O'Donohue, A. \& Fisher, J. (2012) Cognitive Behavior Therapy [Terapia de comportamiento cognitivo]. John Wiley \& Sons, Inc.

Piazza, M. \& Fiestas, F. (2014). Prevalencia anual de trastornos y uso de servicios de salud mental en el Perú: Resultados del estudio mundial de salud mental, 2005. Revista Peruana de Medicina Experimental y Salud Publica, 31(1), 30-38. https://bit.ly/3aMrC6K

Ruiz, A., Díaz, M. \& Villalobos, A. (2012). Manual de técnicas de intervención cognitivo conductuales. Desclée de Brouwer.

Sapp, M. (2004). Cognitive-Behavior Theories of Counseling. Traditional and non- traditional approaches [Teorías cognitivo conductuales de la consejería. Enfoques tradicionales y no tradicionales]. Charles C. Thomas.

Sandi, V., M. (2019). "Desensibilización sistemática en una mujer adulta con fobia a los gatos". (Segunda especialidad). Universidad Nacional Federico Villarreal, Lima, Perú.

Torres, S., F. (2016). Relación entre la personalidad y el rendimiento académico en estudiantes de sexto de primaria, segundo y cuarto de secundaria de un colegio de Lima. (Tesis de Licenciatura). Universidad Peruana Cayetano Heredia, Lima, Perú.

Vargas, C., Z. (2009). La investigación aplicada: una forma de conocer las realidades con evidencia científica. Revista educación, 33 (1), 155-165. https://www.redalyc.org/pdf/440/44015082010. pdf

Fecha de recepción: 8 de setiembre 2021

Fecha de aceptación: 20 de octubre 2021 